\title{
Results of Microfracture in the Osteoarthritic Knee with Focal Full-Thickness Articular Cartilage Defects and Concomitant Medial Meniscal Tears
}

\author{
Jae Jeong Lee, MD, Seung Joo Lee, MD, Tae Jin Lee, MD, Tae Hwan Yoon, MD, and Chong Hyuk Choi, MD \\ Department of Orthopedic Surgery, Yonsei University College of Medicine, Seoul, Korea
}

\begin{abstract}
Purpose: To evaluate the efficacy of arthroscopic microfracture in patients with focal full-thickness cartilage defects in the osteoarthritic knee.
Materials and Methods: Seventy-six patients were enrolled in this study. They were divided into group I ( $\mathrm{n}=38$ ) who underwent microfracture plus meniscectomy and group II ( $\mathrm{n}=38$ ) who underwent only meniscectomy. Clinical and radiological evaluations were performed.

Results: At the time of the three-year follow-up, a total of five failures (6.6\%) were reported: four patients in group I and one in group II. The two groups showed no significant difference in the Lysholm score, the Tegner activity score and the visual analog pain scale score at three years after surgery. However, at the time of the three-month follow-up, group II showed significantly more improvement in the Tegner activity and the visual analog pain score compared with group I.

Conclusions: In the osteoarthritic knee, additional microfracture did not confer any additional benefit to meniscectomy.
\end{abstract}

Keywords: Knee, Cartilage, Osteoarthritis, Microfracture

\section{Introduction}

Articular cartilage damage is observed in at least $60 \%$ of patients undergoing an arthroscopic knee procedure ${ }^{1,2)}$, and appear as a focal traumatic osteochondral defect, an osteochondritis dissecans lesion, an early isolated degenerative lesion, or diffuse degenerative disease ${ }^{3)}$. Surgical management for an early isolated degenerative articular cartilage defect of the knee presents a significant challenge to orthopedic surgeons. Many surgical procedures have been developed to treat articular cartilage lesions

Received November 24, 2012; Revised (1st) January 8, 2013;

(2nd) April 3, 2013; Accepted April 9, 2013

Correspondence to: Chong Hyuk Choi, MD

Department of Orthopedic Surgery, Gangnam Severance Hospital, Yonsei University College of Medicine, 211 Eonju-ro, Gangnam-gu, Seoul 135-720, Korea

Tel: +82-2-2019-3415, Fax: +82-2-573-5393

E-mail: choi8422@yuhs.ac

This is an Open Access article distributed under the terms of the Creative Commons Attribution Non-Commercial License (http://creativecommons.org/licenses/by-nc/3.0/) which permits unrestricted non-commercial use, distribution, and reproduction in any medium, provided the original work is properly cited. of the knee, including abrasion arthroplasty ${ }^{4}$, osteochondral autografting $^{5,6)}$, autologous chondrocyte implantation ${ }^{7}$, and microfracture. Microfracture is indicated for focal full-thickness loss of articular cartilage in a weight bearing area between the femur and the tibia or an area of contact between the patella and the trochlea. It is a technically simple, safe, and cost-effective treatment option for focal articular cartilage lesions of the knee. Although this technique may provide practical advantages for the surgeon, the published clinical outcomes are limited to young patients with traumatic focal defects ${ }^{2,89}$. For cartilage defects of an osteoarthritic knee, unicompartmental or total knee arthroplasty (TKA) may demonstrate predictably favorable results. However, these options may not be appropriate for a patient who wants to maintain a high level of athletic activity. Thus, when a focal fullthickness cartilage defect is accidentally found in a patient with osteoarthritis while undergoing an arthroscopic procedure for a meniscal lesion or a loose body, a treatment decision needs to be made for the cartilage defect. Most surgeons have not performed microfracture for cartilage lesions in the osteoarthritic knee when diffuse, multiple, or large lesions are found on the joint surface. However, treatment options for small and focal lesions vary according to the surgeon's preference. In this situation, 
microfracture could be used as an additional procedure, and some investigators reported that arthroscopic microfracture can consistently provide significant symptomatic and functional improvement in the osteoarthritic knee $\mathrm{e}^{8-10)}$.

The aim of the present study was to evaluate the efficacy of arthroscopic microfracture that was performed concurrently with meniscectomy in the osteoarthritic knee with focal full-thickness cartilage defects based on comparisons with a control group.

\section{Materials and Methods}

Our study population consisted of 76 patients (17 men and 59 women) with the mean age of 60.6 years (range, 50 to 74 years). All patients underwent partial $(\mathrm{n}=60)$ or subtotal $(\mathrm{n}=16)$ meniscectomy. The follow-up period was a minimum of 36 months (mean, 42 months; range, 36 to 50 months).

Group I included 38 osteoarthritis patients who presented with symptomatic medial meniscus tears and focal grade IV full-thickness articular cartilage lesions of the medial femoral condyle categorized according to the Outerbridge classification ${ }^{11}$ between May 2004 and June 2006. Medial meniscectomy and microfracture on the medial femoral condyle were performed by the senior author in these 38 patients. To compare the results of group I, a matched-group analysis was performed with 38 osteoarthritis patients (group II) who were selected from more than 100 patients who had undergone only medial menisectomy for medial meniscal tears accompanied with a focal full-thickness cartilage defect in the medial femoral condyle between 2002 and 2006. Matching parameters were age (within 3 years), gender, length of follow-up, type of meniscectomy (partial or subtotal), and the surgeon. For both groups, the inclusion criterion was Kellgren-Lawrence radiographic grade ${ }^{12)}$ II or III osteoarthritis, and the indications for surgery were as follows: persistent mechanical meniscal pain ${ }^{13)}$ despite six months of conservative treatment which consisted of medication and physical therapy, a clear meniscal sign (positive McMurray test or joint line tenderness), the absence of varus thrust in walking, and the mechanical axis lying across the lateral half of the tibia plateau on the standing radiograph of the whole lower limbs (hip to ankle). Exclusion criteria included multiple chondral lesions or diffuse chondral lesions, traumatic chondral lesions, severe osteoarthritis (Kellgren-Lawrence grade 4), severe varus axis, ligament instability, and inability to follow the rehabilitation protocol.

Clinical assessment was performed retrospectively using the arthroscopic surgery database, medical records, and telephone interviews. Clinical outcome was evaluated using the Lysholm score $^{14)}$, the Tegner activity scale ${ }^{15)}$ and visual analog scale (VAS)grading of knee pain. On the 100-mm VAS, scores (0-100) for pain (0, no pain; 100 , worst possible pain $)^{16,17)}$ were recorded. Patients were evaluated before surgery and at 3 months, 12 months, and 36 months after surgery. A failure was defined as a case requiring a new surgical procedure for the same knee during the follow-up period. Radiographic evaluation included a standing weight-bearing anteroposterior (AP) view and a fulllength AP view. The radiographs were classified according to the Kellgren and Lawrence grading system. AP radiographs were taken while the patient extended the knee joint and evenly distributed the body weight on both lower extremities. For evaluation of the age effect, patients who did not have a failure were assigned to four different groups (group I: young 15 patients, old 19 patients; group II: young 16 patients, old 21 patients) by their age (50-59 years or 60-74 years) and treatment (microfracture and observation).

\section{Surgical Technique}

Patients of both groups underwent arthroscopic meniscectomy. The torn meniscal region that was unstable was selectively removed, and the stable parts were saved as much as possible.

In group I, the microfracture procedure was performed as described by Steadman et al. ${ }^{18)}$. The focal lesion was debrided down to the subchondral bone and laterally to the healthy surrounding cartilage with the use of arthroscopic shavers. The area of the lesion was calculated as square millimeters by measuring the length and width using a meniscal probe ${ }^{19)}$. Angled awls were used to make holes through the subchondral bone plate approximately 3 to $4 \mathrm{~mm}$ apart. Marrow elements accessed by the subchondral bone could be seen as they essentially came from all microfracture holes after the inflow was stopped. The same postoperative pain medication was administered to both groups. The postoperative rehabilitation protocol after microfracture plays a critical role in optimizing outcomes. After surgery, patients were placed in a continuous passive motion machine (CPM) set at 30-70 degrees and a rate of one cycle per minute. The CPM was used for 6-8 hour per day for eight weeks. Weight bearing was not permitted during the initial 8-week healing phase. After completion of the initial healing phase, patients progressed to weight bearing as tolerated, and most of the patients weaned off crutches over the period of one week. And we suggested patients to do closed chain exercises as tolerated for another eight weeks. A detailed description is well documented in a study by Yen et al. ${ }^{9)}$. 


\section{Statistical Analyses}

The statistical analyses were performed using the SAS ver. 9.3 (SAS Institute Inc., Cary, NC, USA). Descriptive statistics, including means, standard deviations, and minimum and maximum values were calculated for every group. Repeated measures analysis of variance (ANOVA) was used to determine whether there were significant differences between both groups. If there were significant differences, a post-hoc test with the Bonferroni adjustment was used to determine which time points were significantly different from each other. The level of significance for all statistical tests was established as $\mathrm{p}<0.05$.

\section{Results}

Four patients were not available for examination in the outpatient clinic. However, these patients (none of whom had a failure) were contacted by telephone, and they answered a questionnaire.

Medial meniscus tears were posterior horn or root tear in all patients and treated with partial or subtotal meniscectomy. The mean size of the medial femoral cartilage lesion was $170 \mathrm{~mm}^{2}$ (100 to $320 \mathrm{~mm}^{2}$ ), and the lesions were located on the weight bearing dome in the range of 10 to 100 degree knee flexion. No significant difference between the treatment groups was found in the defect size. Before surgery, the treatment groups showed no significant difference in the Lysholm score, the Tegner activity scale, the VAS score, and Kellgren-Lawrence grade.

At the time of the three-year follow-up, a total of five failures (6.6\%) were reported: four in group I (10.5\%) and one in group II (2.6\%). The mean time from arthroscopy to TKA was 28.8 months (range, 18 to 48 months).

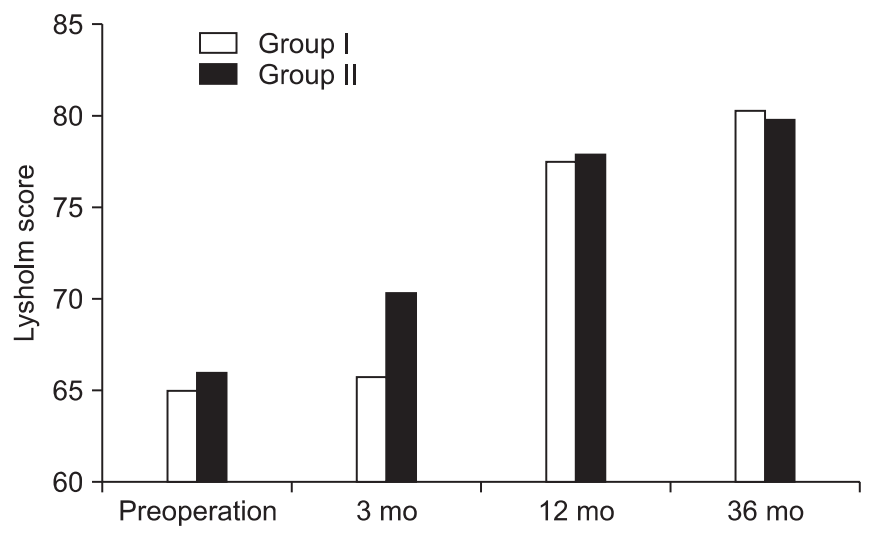

Fig. 1. Bar graph showing the Lysholm scores from before operation to the last follow-up point. The postoperative 3-year Lysholm scores revealed no significant difference between the treatment groups $(\mathrm{p}=0.503)$. Group I: microfracture (white), Group II: observation (black).
Clinical data on patients without failure were collected until the 3rd postoperative year. The mean Lysholm score, the mean Tegner activity scale, and the mean VAS score for pain improved significantly $(\mathrm{p}<0.001)$ for the three postoperative years in both groups. In group I, 27 patients (79.4\%) showed improved Lysholm scores after the operation, two patients (5.9\%), no change, and five patients (14.7\%), a reduction in the Lysholm score. The mean score increased from 65.1 before surgery to 80.3 after surgery $(\mathrm{p}<0.001)$. After the surgery, the Tegner activity score was improved in 27 patients (79.4\%), showed no change in five patients (14.7\%), and dropped in two patients (5.9\%). The mean score increased significantly from 2.9 to 4.2 $(\mathrm{p}<0.001)$. Patients were assessed using the VAS scoring system pre- and postoperatively. In this assessment, 25 patients (73.5\%) reported pain reduction, seven patients (20.6\%), no change, and two patients (5.9\%), an increase in pain. The average VAS score decreased from 68.2 preoperatively down to 23.8 at the last follow-up $(\mathrm{p}<0.001)$. Compared with baseline values, $82.1 \%$ of group II improved on the Lysholm score, $71.8 \%$ showed an improvement on the Tegner activity scale, and $89.7 \%$ had less pain.

However, there was no significant difference in the Lysholm score from baseline to the 3rd postoperative year between the two groups (Fig. 1). No significant difference between the treatment groups was found in the Tegner activity scale (Fig. 2), VAS score for pain (Fig. 3) at one year and three years after treatment. However, at the time of three months follow-up, group II showed significantly more improvement in the Tegner activity scale $(\mathrm{p}=0.004)$ and the VAS score for pain $(\mathrm{p}<0.001)$ compared with group I. There was a tendency toward low Lysholm score in the microfracture group at the $3 r d$ postoperative month, but there

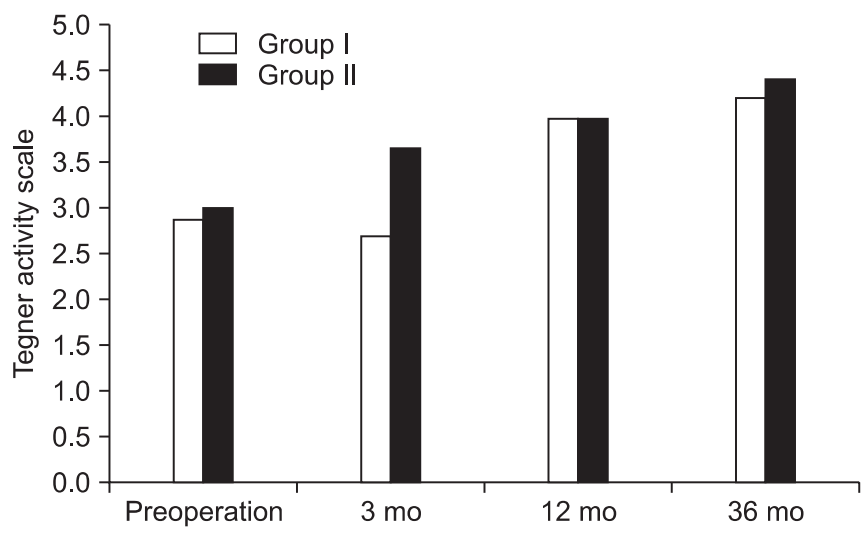

Fig. 2. Bar graph showing the Tegner activity scale from before operation to the last follow-up point. The postoperative 3-year Tegner activity scale revealed no significant difference between the treatment groups ( $\mathrm{p}=0.95)$. Group I: microfracture (white), Group II: observation (black). 


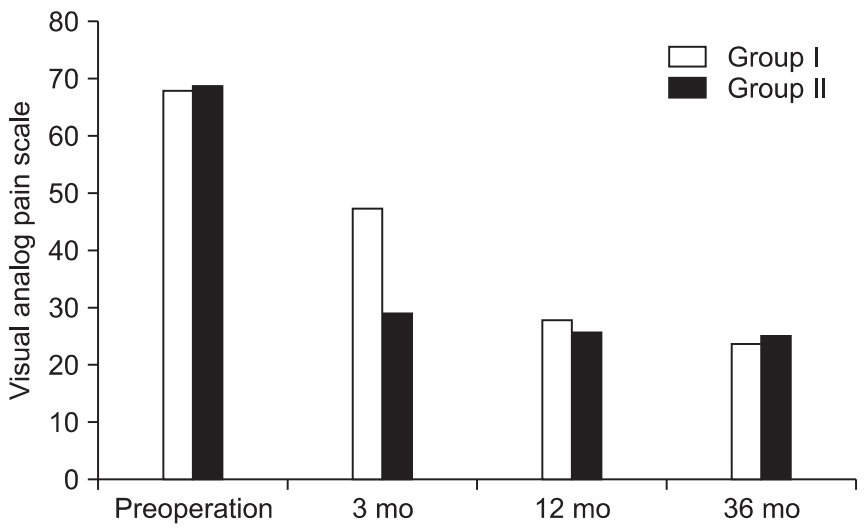

Fig. 3. Bar graph showing the visual analog pain scale from before operation to the last follow-up point. The postoperative 3-year visual analog pain scale revealed no significant difference between the treatment groups $(\mathrm{p}=0.856)$. Group I: microfracture (white), Group II: observation (black).

was no significant difference. However, this tendency was not found one year and three years after surgery. Also, at three years after treatment, younger patients (below 60 years old) showed better VAS score for pain than did older patients, regardless of their treatment group.

Of the total 71 patients who completed radiologic examinations and did not have a failure, grade 2 Kellgren-Lawrence radiographic changes were present in four patients in group I and in five patients in group II at three years after treatment. Grade I Kellgren-Lawrence radiographic changes were present in 19 patients in group I and in 17 patients in group II at three years after treatment. However, no significant difference was found between the two groups regarding the frequency of radiographic changes at the 3 rd postoperative year.

\section{Discussion}

A consensus has not been reached for optimal treatment options for osteoarthritis with focal full-thickness chondral lesions. Although arthroscopic lavage and debridement of the arthritic knee have provided some pain relief, this benefit is usually temporary ${ }^{19-21)}$. Miller et al. ${ }^{10)}$ and Stedman et al. ${ }^{22,23)}$ has reported good clinical outcomes after microfracture in patients aged between 13 and 78 years with the total treated area ranging from 20 to 2,000 $\mathrm{mm}^{2}$ (with mean postoperative Lysholm score ranging from 74 to 89 ). However, it would seem reasonable to believe that a small lesion in a young individual tend to heal better than a large lesion in an old individual after microfracture procedure because the evolving repair tissue is better protected and the regeneration power and quality of fibrous cartilage are better in the former case. Kreuz et al. ${ }^{24)}$ found better clinical outcomes in patients aged 40 years or younger compared to patients over 40 years after microfracture for full-thickness defects ranging from 100 to $400 \mathrm{~mm}^{2}$ in size. Similarly, Knutsen et al. ${ }^{25)}$ reported better results at two years after microfracture in patients with the age of less than 30 years or defects smaller than $400 \mathrm{~mm}^{2}$.

However, Miller et al. ${ }^{10)}$ reported unfavorable outcomes of microfracture in 81 patients with degenerative knees. The average patient age was 49 years (range, 40 to 70 years), and the average size of degenerative lesion was $229.5 \mathrm{~mm}^{2}$ (range, 25 to $2,000 \mathrm{~mm}^{2}$ ). The average time of follow-up was 2.6 years (range, 2 to 5 years). Their mean Lysholm score improved from preoperative 53.8 to postoperative 83.1, and the mean Tegner score, from 2.9 to 4.5. In 2006, Bae et al. ${ }^{8}$ reported that patients with full-thickness chondral defects in the osteoarthritic knee could obtain functional improvement and an increase in the joint space after microfracture. They reported that the joint space was widened by $1.06 \mathrm{~mm}$ on standing anteroposterior radiographs and by $1.37 \mathrm{~mm}$ on standing lateral radiographs. Also, Yen et al. ${ }^{9)}$ reported that microfracture is a cartilage restoration technique that has proven to provide clinical benefits in the osteoarthritic knee, and it is a relatively simple procedure that can be concurrently performed with other arthroscopic procedures and require minimal equipment. They concluded that it is vital to select appropriate patients who have minimal malalignment and ability to adhere to the postoperative regimen, and the success of microfracture depends upon surgical technique and rehabilitation after surgery. Based on the evidence of the above studies, microfracture has been used as an incidental procedure for accidentally discovered small articular defects in the osteoarthritic knee. However, fibrocartilaginous tissue produced by microfracture technique does not have the biomechanical properties and durability of the original articular cartilage and the treatment probably does not alter the progression of osteoarthritis. Therefore, microfracture is not a curative treatment; however, symptom relief has been observed in many patients over the several years. It has been reported that the results are good for small and focal chondral lesions that occur in patients with moderate osteoarthritis ${ }^{8,9,20)}$. Unfortunately, no randomized or controlled prospective studies have appropriately evaluated this technique for osteoarthritic patients. So, the use of bone marrow stimulation techniques in patients with knee osteoarthritis has not been validated yet.

We found no significant differences in the clinical and 
radiological results between groups I and II at the last followup after adjusting for pretreatment values. At three months after surgery, the microfracture group tended to score low on the Tegner activity scale and high on the VAS for pain. This difference in clinical outcome may be attributed to weakness in the quadriceps muscle due to nonweight bearing rehabilitation in microfracture group. Patients undergoing only menisectomy are generally allowed to return to their normal activities almost immediately after surgery. Patients undergoing both microfracture and menisectomy adhered to a strict postoperative rehabilitation regimen for eight weeks after surgery. There is an advantage in group II patients who return to their everyday activities sooner. Because no significant differences were seen in clinical and radiological results between the groups at the last follow-up, in the setting of osteoarthritis, arthroscopic microfracture did not confer any additional benefit to menisectomy in medial meniscus tear patients with a focal full-thickness cartilage defect on the medial femoral condyle.

In our present study, the success rate of group I with microfracture was $79.4 \%$ in terms of the Lysholm score. A similar success rate has been reported before. In a study by Steadman et al. ${ }^{26}, 80 \%$ of their patients rated their condition as improved seven years after microfracture surgery. All these patients were under 45 years of age when they were included in the study. Steadman et al. ${ }^{23)}$ also reported a failure rate of $13 \%$ in 61 patients (69 knees) with radiographically verified osteoarthritis treated with debridement and microfracture. In this present study, five patients (6.25\%) required total knee replacement after an average of 28.8 months: four patients in microfracture group and one patient in observation group. Although no significance was found due to the small number of failure cases, our hypothesis for higher number of failure cases in group I was that microfracture could lead to destruction of subchondral bone integrity that is important for sustaining load bearing.

The strengths of our study are the inclusion of a control group and the use of patient-administered outcome scores. Our study is the first to investigate the effect of microfracture on cartilage defects in osteoarthritis based on comparisons with a control group.

The present study indicates that even though we accidentally found focal cartilage defects in early osteoarthritis patients, arthroscopic microfracture surgery provided no additional benefit. Since patients over 60 years old showed particularly poor results after the procedure, we do not recommend arthroscopic microfracture to be used as an additional procedure for focal fullthickness cartilage defects in osteoarthritis patients.
We recognize the limitations of our present study. Important limitations of our study are that we do not have the data on pure cartilage defect symptoms, and it is difficult to distinguish the results of microfracture from the effects of menisectomy for meniscal symptoms. Other weaknesses of our study are the small number of patients, short follow-up period, retrospective collection of data, and the fact that neither a routine second-look arthroscopy nor an MRI examination was performed.

\section{Conclusions}

We conclude that in early osteoarthritis patients with symptomatic medial meniscus tears, arthroscopic microfracture surgery for a focal full-thickness cartilage defect on the medial femoral condyle did not confer any additional benefit to meniscectomy.

\section{Conflict of Interest}

No potential conflict of interest relevant to this article was reported.

\section{References}

1. Curl WW, Krome J, Gordon ES, Rushing J, Smith BP, Poehling GG. Cartilage injuries: a review of 31,516 knee arthroscopies. Arthroscopy. 1997;13:456-60.

2. Hjelle K, Solheim E, Strand T, Muri R, Brittberg M. Articular cartilage defects in 1,000 knee arthroscopies. Arthroscopy. 2002;18:730-4.

3. Sgaglione NA, Chen E, Bert JM, Amendola A, Bugbee WD. Current strategies for nonsurgical, arthroscopic, and minimally invasive surgical treatment of knee cartilage pathology. Instr Course Lect. 2010;59:157-80.

4. Childers JC Jr, Ellwood SC. Partial chondrectomy and subchondral bone drilling for chondromalacia. Clin Orthop Relat Res. 1979;(144):114-20.

5. Bobic V. Arthroscopic osteochondral autograft transplantation in anterior cruciate ligament reconstruction: a preliminary clinical study. Knee Surg Sports Traumatol Arthrosc. 1996;3:262-4.

6. Insall JN. Intra-articular surgery for degenerative arthritis of the knee: a report of the work of the late K. H. Pridie. J Bone Joint Surg Br. 1967;49:211-28.

7. Brittberg $\mathrm{M}$, Lindahl A, Nilsson A, Ohlsson C, Isaksson O, Peterson L. Treatment of deep cartilage defects in the knee with autologous chondrocyte transplantation. N Engl J Med. 
1994:331:889-95.

8. Bae DK, Yoon KH, Song SJ. Cartilage healing after microfracture in osteoarthritic knees. Arthroscopy. 2006;22:36774.

9. Yen YM, Cascio B, O’Brien L, Stalzer S, Millett PJ, Steadman JR. Treatment of osteoarthritis of the knee with microfracture and rehabilitation. Med Sci Sports Exerc. 2008;40:200-5.

10. Miller BS, Steadman JR, Briggs KK, Rodrigo JJ, Rodkey WG. Patient satisfaction and outcome after microfracture of the degenerative knee. J Knee Surg. 2004;17:13-7.

11. Outerbridge RE. The etiology of chondromalacia patellae. J Bone Joint Surg Br. 1961;43:752-7.

12. Kellgren JH, Lawrence JS. Radiological assessment of osteoarthrosis. Ann Rheum Dis. 1957;16:494-502.

13. Bin SI, Kim JM, Shin SJ. Radial tears of the posterior horn of the medial meniscus. Arthroscopy. 2004;20:373-8.

14. Kocher MS, Steadman JR, Briggs KK, Sterett WI, Hawkins RJ. Reliability, validity, and responsiveness of the Lysholm knee scale for various chondral disorders of the knee. J Bone Joint Surg Am. 2004;86:1139-45.

15. Tegner Y, Lysholm J. Rating systems in the evaluation of knee ligament injuries. Clin Orthop Relat Res. 1985;(198):43-9.

16. Ageberg E, Roberts D, Holmstrom E, Friden T. Balance in single-limb stance in patients with anterior cruciate ligament injury: relation to knee laxity, proprioception, muscle strength, and subjective function. Am J Sports Med. 2005;33:1527-35.

17. Borsa PA, Lephart SM, Irrgang JJ. Comparison of performance-based and patient-reported measures of function in anterior-cruciate-ligament-deficient individuals. J Orthop Sports Phys Ther. 1998;28:392-9.

18. Steadman JR, Rodkey WG, Briggs KK. Microfracture to treat full-thickness chondral defects: surgical technique, rehabilitation, and outcomes. J Knee Surg. 2002;15:170-6.

19. Edelson R, Burks RT, Bloebaum RD. Short-term effects of knee washout for osteoarthritis. Am J Sports Med. 1995;23: 345-9.

20. Moseley JB Jr, Wray NP, Kuykendall D, Willis K, Landon G. Arthroscopic treatment of osteoarthritis of the knee: a prospective, randomized, placebo-controlled trial: results of a pilot study. Am J Sports Med. 1996;24:28-34.

21. Rand JA. Role of arthroscopy in osteoarthritis of the knee. Arthroscopy. 1991;7:358-63.

22. Steadman JR, Miller BS, Karas SG, Schlegel TF, Briggs KK, Hawkins RJ. The microfracture technique in the treatment of full-thickness chondral lesions of the knee in National Football League players. J Knee Surg. 2003;16:83-6.

23. Steadman JR, Ramappa AJ, Maxwell RB, Briggs KK. An arthroscopic treatment regimen for osteoarthritis of the knee. Arthroscopy. 2007;23:948-55.

24. Kreuz PC, Erggelet C, Steinwachs MR, Krause SJ, Lahm A, Niemeyer P, Ghanem N, Uhl M, Sudkamp N. Is microfracture of chondral defects in the knee associated with different results in patients aged 40 years or younger? Arthroscopy. 2006;22:1180-6.

25. Knutsen G, Drogset JO, Engebretsen L, Grøntvedt T, Isaksen V, Ludvigsen TC, Roberts S, Solheim E, Strand T, Johansen O. A randomized trial comparing autologous chondrocyte implantation with microfracture: findings at five years. J Bone Joint Surg Am. 2007;89:2105-12.

26. Steadman JR, Briggs KK, Rodrigo JJ, Kocher MS, Gill TJ, Rodkey WG. Outcomes of microfracture for traumatic chondral defects of the knee: average 11-year follow-up. Arthroscopy. 2003;19:477-84. 